\title{
Laterality of Skin Temperature and Magnetic Resonance Imaging in Patients with Wallenberg Syndrome
}

\author{
Sun Ki Min, MD* (D); Jinyoung Oh, MD* (D); Taemin Kim, MD* (D); Ji Eun Han, MD* (D); Sang Won Han, MD* (D); \\ Hyun-jeung Yu, MD, $\mathrm{PhD}^{\dagger}$ \\ Department of Neurology, Sanggye Paik Hospital, Inje University College of Medicine, Seoul; Department of Neurology, Bundang Jesaeng \\ General Hospital ${ }^{\dagger}$, Seongnam, Korea
}

Background: Recently, lateral differences in body surface temperature (BST) have been reported as a symptom of Wallenberg syndrome (WS), resulting from disturbances in the sympathetic nerve pathway. This study aimed to investigate the relationship between the laterality of BST and brain magnetic resonance imaging (MRI) findings in 12 patients with WS.

Methods: BST was measured using an infrared thermal camera at $7 \pm 3$ days and $90 \pm 30$ days after symptom onset. The MRI findings were categorized as rostral, middle, and caudal medulla rostrocaudally and typical, ventral, large, dorsal, and lateral types in the horizontal direction.

Results: MRI revealed medullary lesions on the right in five patients and on the left in seven patients. Two patients without lateralized BST had lateral caudal medullary infarction, and one patient had a dorsal middle medullary infarction. One patient with lateralized BST had a rostral medullary infarction and the other had a typical or large middle medulla infarction. Lateralized BST in patients with WS may disturb the sympathetic nervous system pathway that descends from the rostral ventrolateral medulla oblongata. Deficits in sweating and skin blood flow may cause BST laterality.

Conclusion: This study showed that lateralized BST in patients with WS may be associated with disturbances in the sympathetic nervous pathway descending from the rostral ventrolateral medulla. These results support the assumption that autonomic dysfunction may be related to abnormal sensory symptoms in patients with WS.

\section{J Neurosonol Neuroimag 2021;13(2):47-54}

Key Words: Lateral medullary syndrome; Magnetic resonance imaging; Temperature; Thermography
Received: August 17, 2021

Revised: September 27, 2021

Accepted: September 30, 2021

Correspondence:

Hyun-jeung Yu, MD, PhD

Department of Neurology, Bundang Jesaeng General Hospital, 20 Seohyeon-ro 18obeon-gil,

Bundang-gu, Seongnam 1359o, Korea

Tel: +82-31-779-0216

Fax: +82-31-779-0897

E-mail:yhj314@dmc.or.kr

\section{INTRODUCTION}

Wallenberg syndrome (WS) is caused by dorsal lateral medullary infarction (LMI) that results from occlusion of the vertebral artery or posterior inferior cerebellar artery (PICA). ${ }^{1}$ Clinical symptoms of WS include hoarseness, dysphagia, sensory disturbance, vertigo, ataxia, and Horner's syndrome. ${ }^{2,3}$ Recently, the lateral difference in body surface temperature (BST) has been reported as a symptom of WS, resulting from disturbances of the sympathetic nerve pathway. ${ }^{4,5}$ We have previously reported the laterality of skin temperature depending on sensory symptoms in patients with WS. ${ }^{6,7}$ Our study showed that patients with sensory dysfunction in WS had lateral BST differences, which were detected by infrared thermography. We presumed that the laterality of BST and sensory dysfunction in patients with WS may be associated with the distur- 
bance of the cutaneous vasomotor activity of skin blood flow descending from the rostral ventrolateral medulla oblongata (RVLM). ${ }^{6}$ This study aimed to investigate the relationship between the laterality of BST and brain magnetic resonance imaging (MRI) findings in 12 patients with WS.

\section{SUBJECTS AND METHODS}

The methods of this study have been published in a previous study. ${ }^{6}$ In brief, patients were eligible if they were $\geq 20$ years old and had their first LMI within 7 days of symptom onset. Patients with recurrent cerebral infarction, ankle-bracing index $<0.9$, or abnormal autonomic function tests were excluded from this study.

The patients' accompanying vascular risk factors, laboratory tests, BST, and brain MRI were assessed at admission. BST was measured using an infrared thermal camera (SMART T-1000; MESH Co, Wonju, Korea) at $7 \pm 3$ days and $90 \pm 30$ days after the onset of symptoms (Fig. 1). The BST in this study was not a true skin surface temperature but a noncontact temperature for lateral comparison. BST was analyzed at four locations (the nasolabial fold on the face, $5 \mathrm{~cm}$ above the umbilicus, and anterior forearm and shin) bilaterally. Laterality was defined as significant when the BST was macroscopically different, and the discrepancy was more than $0.5^{\circ} \mathrm{C}$ between the right and left sides. ${ }^{5}$ An experienced researcher performed all BST measurements, and throughout the trial, the researcher was blinded to the clinical symptoms of the patients.

Three neurologists blinded to the thermography results classified the patients' MRI findings according to the well-known LMI classification system (Fig. 2). ${ }^{1}$ Briefly, the lesions were categorized as rostral, middle, and caudal medulla rostrocaudally and typical, ventral, large, dorsal, and lateral type horizontally. In the case of disagreement in classifying a medullary lesion by the raters, a category that was agreed upon by two of the three raters was considered as the final classification for the analyses.

\section{Statistical analyses}

Descriptive data are expressed as numbers (\%) or means \pm standard deviations. Categorical data between groups were compared using chi-square and Fisher exact tests. The Mann-Whitney $U$ test was used to compare non-normally distributed data, and Student's
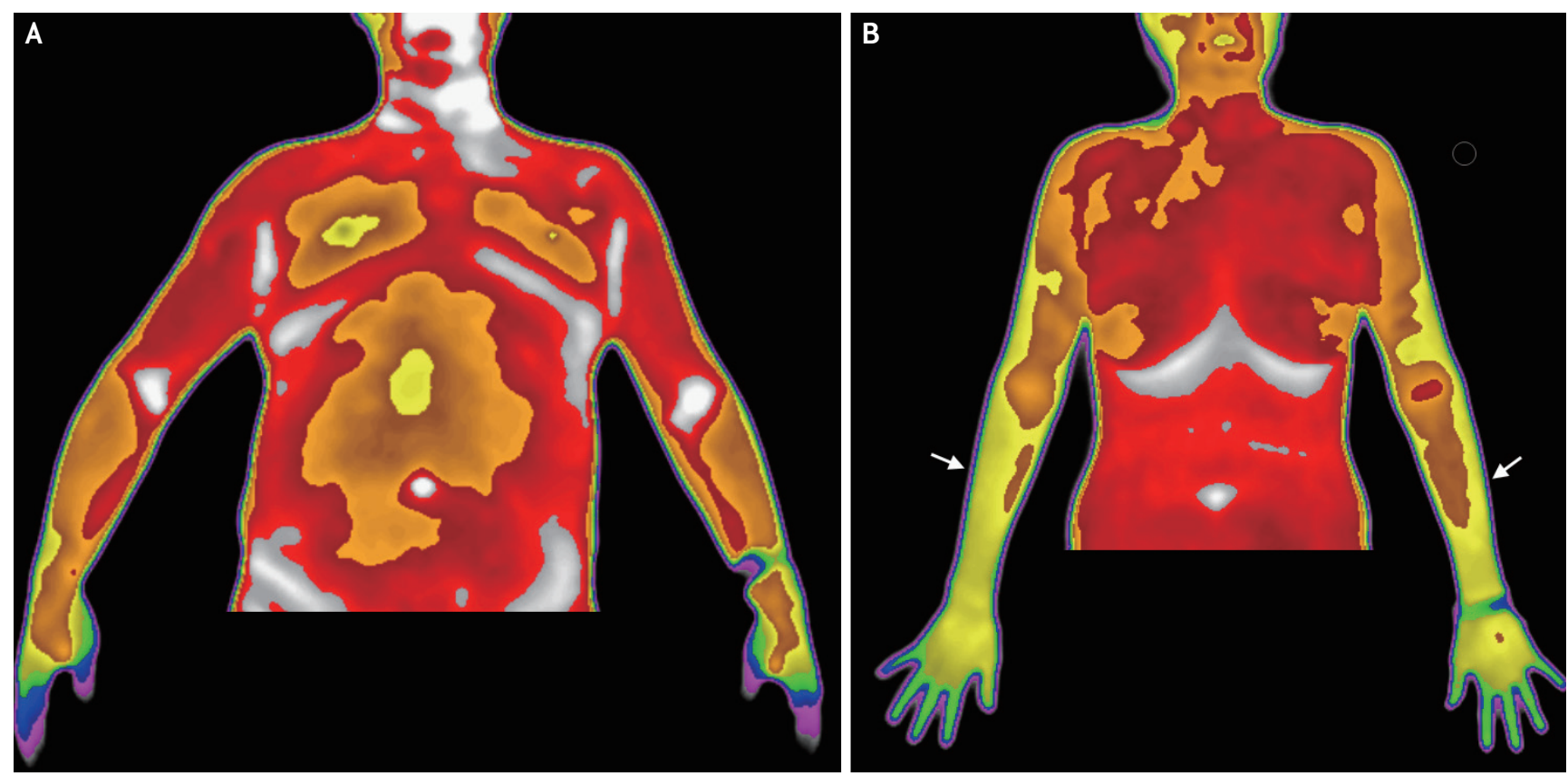

FIG. 1. Thermographic images in patients with Wallenberg syndrome. (A) Symmetric and (B) asymmetric body surface temperature (arrows in anterior forearm). 
$t$-test was used to compare normally distributed data. Two-sided null hypotheses of no difference were rejected if $p$-values were lower than 0.05 . Statistical analyses were performed using SPSS version 25.0 for Windows (IBM Co., Armonk, NY, USA).

\section{RESULTS}

A total of 1,005 patients who had experienced first-ever ischemic stroke between June 2018 and November 2020 were screened. During the trial, 16 patients had WS, of which four refused to participate in the study. The final analyses included 12 patients with WS (10 patients with pure LMI and two patients with concomitant PICA territorial cerebellar infarction). The baseline characteristics of the patients are as follows. There were 10 males and two females, aged 43 to 81 (mean 59.9 \pm 11.9 ) years. Fifty percent of the patients had a history of hypertension, $42.0 \%$ had diabetes, $33.0 \%$ had dyslipid-
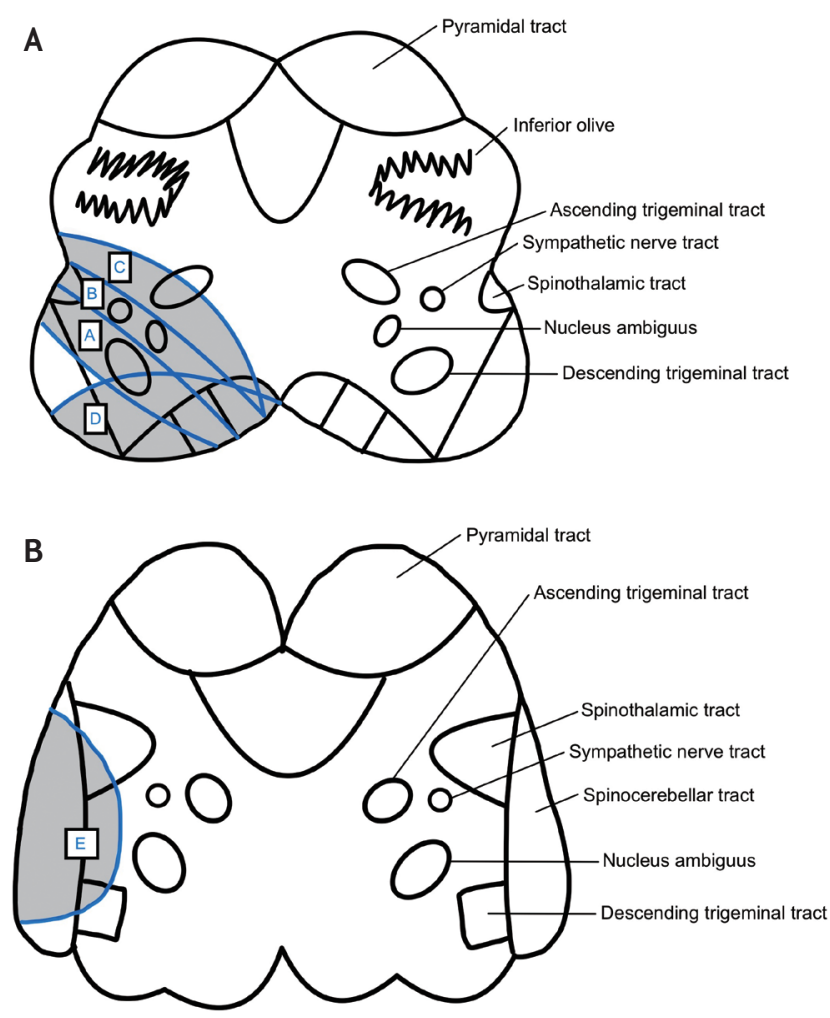

FIG. 2. Diagram of essential medullary structures at the (A) middle and (B) caudal level. Shaded areas specify different patterns of lateral medullary infarction $(A+B$, typical; $B+C$, ventral; $A+B+C$, large; $D$, dorsal; E, lateral). ${ }^{1}$ emia, $8.0 \%$ had coronary artery disease, and $23.0 \%$ were current cigarette smokers. There were no differences in age or sex in the presence of lateral BST differences. The most frequent stroke subtype was a stroke of undetermined etiology, negative workup (41.6\%), followed by cardioembolism (16.7\%), lacunes (16.7\%), vertebral artery dissections (16.7\%), and large artery atherosclerosis (8.3\%).

Tables 1, 2 show the results of the infrared thermography. The median time from symptom onset to conducting thermographic scanning was 8 days (5-10 days) and 93 days (63-115 days), respectively. In the 7-day study, the laterality of BST was not detected in patients without sensory symptoms. In contrast, all patients with sensory symptoms showed laterality of BST (three patients with right side lesion in Table 1, five patients with left side lesion in Table 2). Of these, six patients showed lateralized BST at multiple sites (face, body, upper limb, or lower limb) and warmer ipsilateral to the infarction. Laterality was more common in the upper limb (88.0\%), lower limb (63.0\%), body (63.0\%), and face (13.0\%). Two patients showed laterality of BST only in the upper or lower limb only. Follow-up thermography at 90 days was performed in eight patients. Five patients had residual sensory symptoms and lateralized BST. Laterality was also common in the upper or lower limb (60.0\%) and body (40.0\%), and warmer ipsilateral to the infarction. Lateralized BST was not observed in three patients with no sensory symptoms, suggesting that residual sensory symptoms were related to the laterality of BST.

Brain MRI and diffusion-weighted imaging were performed in all patients. MRI revealed that the medullary lesions were on the right in five patients and on the left in seven patients. In this study, the lesions were categorized rostrocaudally and horizontally according to the LMI classification system. ${ }^{1}$ In the right LMI group, one patient with no lateralized BST (Fig. 3A), had a small caudal medullar infarction (caudal medulla-lateral type) and patients with lateralized BST had a larger middle medullary infarction (middle medulla-typical in Fig. ${ }_{3} \mathrm{~B}-\mathrm{D}$ and large type in Fig. $\left.{ }_{3} \mathrm{E}\right)$. In the left LMI group, two patients with no lateralized BST showed a caudal medulla-lateral type (Fig. 4A) or a middle medulla-dorsal type (Fig. 4B) infarction. Four patients with lateralized BST showed middle medulla-typical (Fig. $4 \mathrm{C}$ ) or 

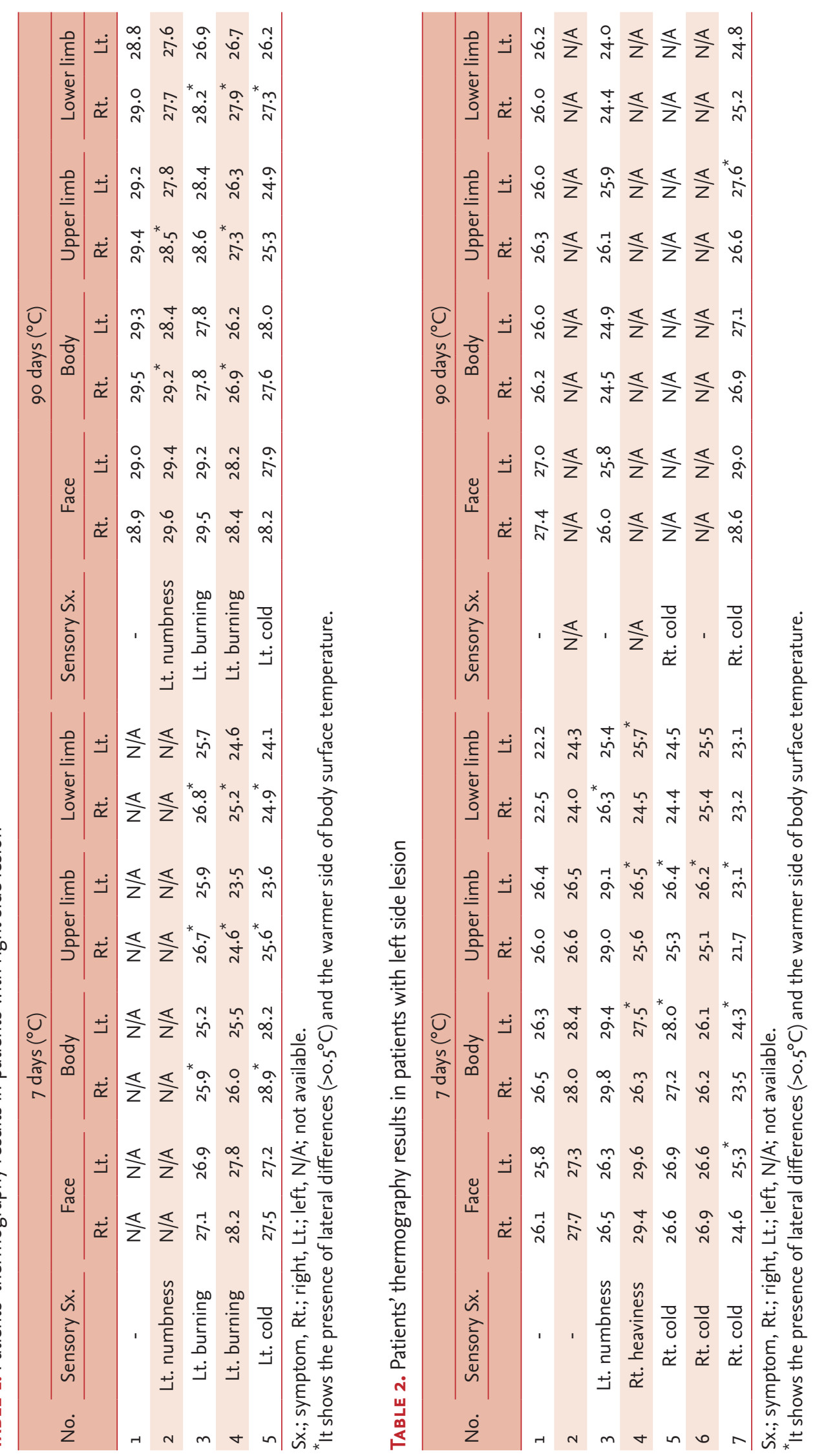

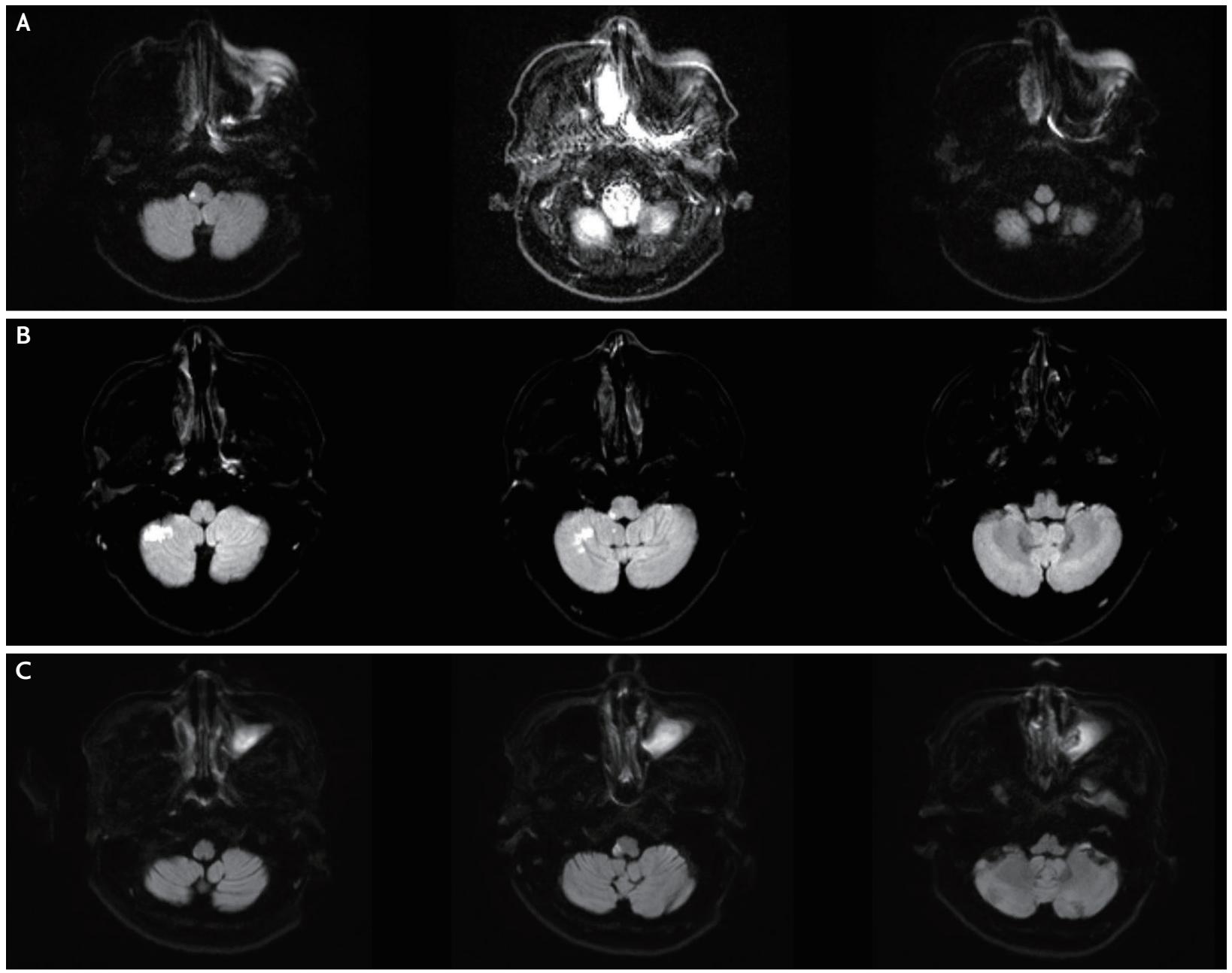

D
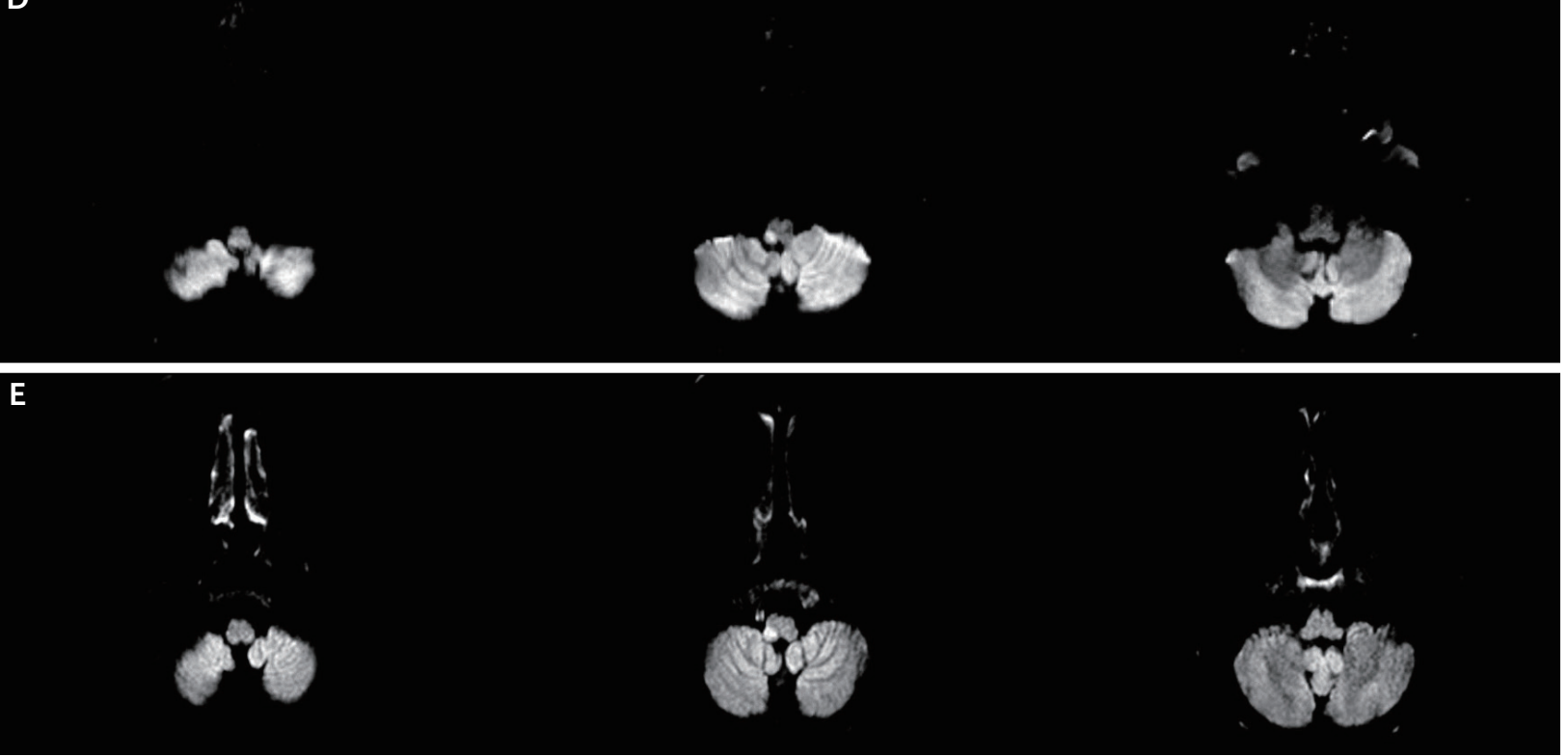

FIG. 3. Brain magnetic resonance imaging findings of the right lateral medullary infarction (LMI). (A) One patient with no lateralized body surface temperature (BST) shows a small caudal medullar infarction (caudal medulla-lateral type) and patients with lateralized BST have a larger middle medullary infarction (middle medulla-typical in [B-D] and large type in [E]). 

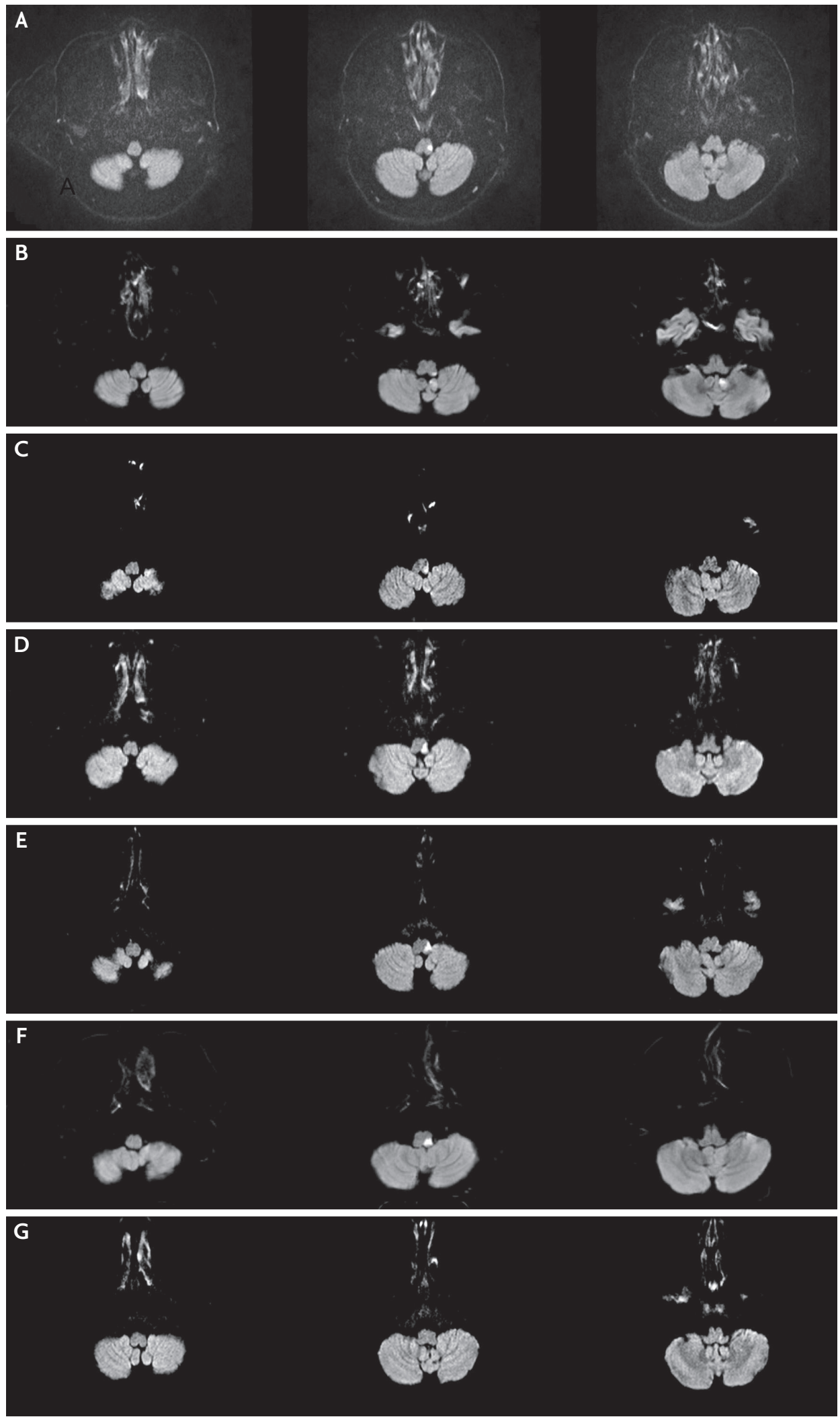

FIG. 4. Brain magnetic resonance imaging findings of the left lateral medullary infarction. Two patients with no lateralized body surface temperature (BST) show (A) a caudal medulla-lateral type or (B) a middle medulla-dorsal type infarction. Four patients with lateralized BST have (C) middle medulla-typical or (D-F) large type infarction. One patient with lateralized BST reveals (G) a rostral medullary infarction. 
large type (Fig. 4D-F) infarction. One patient with lateralized BST showed a rostral medullary infarction (Fig. $\left.{ }_{4} \mathrm{G}\right)$. Taken together, two patients without lateralized BST had a lateral caudal medullary infarction, and one patient had a dorsal middle medullary infarction. One patient with lateralized BST had a rostral medullary infarction and the other had a typical or large middle medulla infarction.

\section{DISCUSSION}

This study explored the relationship between the laterality of BST and brain MRI findings in 12 patients with WS. Our study showed that while patients without lateralized BST had caudal medulla-lateral type or middle medulla-dorsal type LMI, all patients with lateralized BST had a middle medulla-typical or large type, or rostral medullary infarction.

The hypothalamus, which receives information from cutaneous and internal thermoreceptors and sends efferent signals to thermoregulatory effector organs, plays a key role in controlling body temperature. ${ }^{8}$ In the brain stem medulla, the medullary raphe and RVLM are important structures in controlling cutaneous vasomotor activity for thermoregulation. ${ }^{9,10}$ The medullary raphe contains a narrow sheet of cells in the midline of the medulla that extends caudally from a level to the rostral pole of the facial nucleus to the level of the pyramidal decussation. ${ }^{11}$ It has sympathetic premotor neurons that connect to the central neural pathway intermediate thermoregulatory signals to sympathetic preganglionic neurons controlling cutaneous sympathetic vasoconstrictor nerves. ${ }^{9,10}$ The RVLM has sympathetic premotor neurons for influencing the thermoregulatory control of cutaneous vasomotor activity and the blood pressure. ${ }^{9}$ Based on anatomical knowledge, the most plausible explanation for lateralized BST in patients with WS may be the disturbance of the sympathetic nervous system pathway descending from the RVLM. Deficits in sweating and skin blood flow may cause BST laterality.

The strengths of this study were that it prospectively recruited consecutive patients with WS. Unlike traditional retrospective studies, this method can eliminate selection bias. Thermographic scan was performed at the same time points in all patients. As the laterality of BST decreased with residual sensory symptoms and time, ${ }^{5,12}$ this study could provide more clinically important information.

Our study had several limitations. Although our study demonstrated that all patients with sensory symptoms showed lateralized BST, we were unable to conclude whether the BST decreased contralateral to the LMI or increased ipsilateral to the LMI. It has been known that vasomotor autonomic dysregulation is associated with asymmetric BST in stroke patients; however, the results of previous studies are conflicting, with some reporting increased and others decreased BST in contralateral to the infarction. ${ }^{8,13,14}$ It may be related that most of these studies are performed in a small population, and no prospective investigations were conducted. Recent studies have consistently shown a marked decreased in BST contralateral to cerebral infarction. ${ }^{8}$ This phenomenon is related to the paretic limbs in hemispheric infarction and to the presence of the LMI in brain stem infarction. However, one study suggests that the cause of lateralized BST in patients with WS is a disruption of the connecting pathway of sweating and skin blood flow descending from the lateral brainstem, including the ventrolateral medulla. ${ }^{5}$ Disrupted sweating and vasoconstrictive function are likely to increase the BST in the ipsilateral to the infarction in patients with WS. Another study showed that lateralized BST in patients with WS is probably caused by damage to the medullary vasomotor centers. ${ }^{8}$ They reported that the ipsilateral side of the face is warmer and hypohidrosis compared with the other side in patients with LMI and Horner's syndrome. Considering the pathway and function of the sympathetic nervous system in the brainstem medullar, our study suggests that BST may be increased ipsilateral to the infarction, especially in patients with WS. Further studies are warranted to determine whether the lesion side BST decreased or increased with cerebral hemispheric and brainstem infarction. Due to the rarity of LMI, only a small number of patients were recruited during the study period. We were unable to evaluate the clinical factors associated with lateralized BST. These limitations should be considered when interpreting the data.

In conclusion, this study showed that lateralized BST in patients with WS may be associated with disturbanc- 
es in the sympathetic nervous pathway that descends from the RVLM. These results support the assumption that autonomic dysfunction may be related to abnormal sensory symptoms in patients with WS.

\section{Ethics Statement}

Written informed consent was obtained from all participants before enrollment in the study. This study was approved by the Clinical Trial Review Committee of Inje University Sanggye Paik Hospital (Approval No. SGPAIK 2018-01-002) and conducted in accordance with the Good Clinical Practice guidelines and the Declaration of Helsinki.

\section{Availability of Data and Material}

The data that support the findings of this study are available from HJY but restrictions apply to the availability of these data, which were used under license for the current study, and so are not publicly available. Data are however available from the authors upon reasonable request and with permission of HJY.

\section{Acknowledgments}

None.

\section{Sources of Funding}

None.

\section{Conflicts of Interest}

No potential conflicts of interest relevant to this article was reported.

\section{REFERENCES}

1. Kim JS. Pure lateral medullary infarction: clinical-radiological correlation of 130 acute, consecutive patients. Brain. 2003;126:1864-1872.

2. Jeon K, Lee C, Lee K, Choi HS, Kim BS, Ko YJ, et al. Bilater- al PICA territory infarcts of right lateral medulla and left cerebellum via spontaneous common PICA dissection. J Neurosonol Neuroimag. 2019;11:149-153.

3. Kim JS, Lee JH, Lee MC. Patterns of sensory dysfunction in lateral medullary infarction. Clinical-MRI correlation. Neurology. 1997;49:1557-1563.

4. Takahashi M, Shinya A, Choh Y, Itaya S, Inaba A, Orimo S. Portable thermographic screening for detection of acute Wallenberg's syndrome. J Vis Exp. 2019 Sep 19. doi: 10.3791/59330 [Epub ahead of print].

5. Takahashi M, Shinya A, Ito N, Ebina J, Abe K, Inaba A, et al. Utility of thermographic measurements of laterality of body surface temperature to prevent misdiagnosis of acute Wallenberg's syndrome. Brain Behav. 2018;8:eo1040.

6. Han JE, Min SK, Oh J, Kim T, Han SW, Lee WY, et al. Laterality of skin temperature depending on sensory symptoms in patient with Wallenberg syndrome. J Korean Neurol Assoc. 2021;39:150-157.

7. Yoon HR, Han JE, Kim KH, Park JH, Kim JY. Skin temperature asymmetry in patient with lower lateral medullary infarction. J Korean Neurol Assoc. 2018;36:387-389.

8. Korpelainen JT, Sotaniemi KA, Myllylä VV. Asymmetrical skin temperature in ischemic stroke. Stroke. 1995;26:15431547 .

9. Ootsuka Y, Tanaka M. Control of cutaneous blood flow by central nervous system. Temperature (Austin). 2015;2:392-405.

10. Tan CL, Knight ZA. Regulation of body temperature by the nervous system. Neuron. 2018;98:31-48.

11. Lovick TA. The medullary raphe nuclei: a system for integration and gain control in autonomic and somatomotor responsiveness? Exp Physiol. 1997;82:31-41.

12. Neves EB, Vilaça-Alves J, Rosa C, Reis VM. Thermography in neurologic practice. Open Neurol J. 2015;9:24-27.

13. Alfieri FM, Massaro AR, Filippo TR, Portes LA, Battistella LR. Evaluation of body temperature in individuals with stroke. NeuroRehabilitation. 2017;40:119-128.

14. Ivey FM, Gardner AW, Dobrovolny CL, Macko RF. Unilateral impairment of leg blood flow in chronic stroke patients. Cerebrovasc Dis. 2004;18:283-289. 